\section{Exposição ocupacional ao benzeno no Brasil: estimativas baseadas em uma matriz de exposição ocupacional}

\author{
Occupational exposure to benzene in Brazil: \\ estimates based on an occupational \\ exposure matrix

\section{Exposición ocupacional al benceno en Brasil: estimativas basadas en una matriz de exposición ocupacional}

Maria Juliana Moura Corrêa 1 Vilma Sousa Santana 1

\section{Resumo}

Estima-se o número de trabalhadores expostos e a prevalência da exposição ocupacional ao benzeno no Brasil. Por causa da falta de mensurações locais disponíveis para a pesquisa, empregaram-se dados de uma matriz de exposição ocupacional, a Finnish National Job-Exposure Matrix (FINJEM), que contemplam proporções de expostos ao benzeno calculadas com medidas ambientais. No Brasil, o Censo Demográfico de 2010 identificou 86.353.839 trabalhadores ativos e ocupados. Aplicando-se os parâmetros da FINJEM, estimou-se que 7.376 .761 (8,5\%) pertenciam a grupos ocupacionais potencialmente expostos, enquanto 770.212 foram considerados expostos ao benzeno, correspondendo a uma prevalência ponderada por grupo ocupacional de 0,9\%, maior em homens $(1,1 \%)$ que em mulheres (0,6\%). Expostos se concentraram no grupo de Operadores e Mecânicos de Máquinas e Motores (62\%). O número de expostos e a prevalência da exposição ocupacional ao benzeno são elevados mesmo considerando parâmetros da Finlândia, sugerindo a necessidade de monitoramento e controle desse cancerígeno no Brasil.

Exposição Ocupacional; Benzeno; Saúde Ocupacional

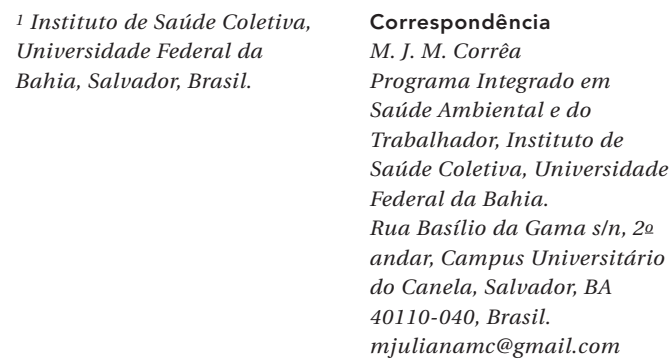




\section{Introdução}

Desde 1982, o benzeno é reconhecido como cancerígeno pela Agência Internacional de Pesquisas sobre o Câncer (IARC) da Organização Mundial da Saúde (OMS), especialmente para tumores do sistema hematopoiético 1 . O benzeno é amplamente utilizado em diversos processos produtivos, como na exploração de petróleo e na indústria petroquímica, e é subproduto de várias indústrias químicas dentre outras. A OMS recomenda normas de controle da exposição ao benzeno, com sua mensuração e monitoramento sistemático e contínuo em ambientes de trabalho, além de medidas de prevenção, que compõem as ações de vigilância em saúde 2 , já em curso em muitos países 3 . Essas ações, entretanto, continuam sendo precariamente implementadas, mesmo em países reconhecidos como avançados na proteção contra riscos químicos. Comumente, mensurações de agentes químicos provêm de pesquisas realizadas com pequenas amostras, limitadas a alguns ramos de atividade econômica ou grupos ocupacionais. Disso resulta, dentre outros problemas, a existência de poucos dados sobre a extensão, gravidade e distribuição dessa exposição ${ }^{3}$.

Tal escassez de informações sobre exposições em ambientes de trabalho tem levado ao uso crescente de matrizes de exposição ocupacional (MEO), instrumentos que permitem calcular ou projetar estimativas, como o número de expostos e a prevalência de exposições, com base em dados parciais e em outros contextos 4. Desde os anos 1980, MEO vêm sendo empregadas para classificar estados e/ou estimar níveis de exposição em trabalhadores, individualmente ou para grupos ocupacionais e ramos de atividade econômica, dentre outros. Sua popularidade é determinada pela rapidez e baixo custo com que informações úteis podem ser geradas ${ }^{5}$. A Finnish National Job-Exposure Matrix (FINJEM) é uma MEO criada na década de 1990 pelo Finnish Institute of Occupational Health (FIOH), cuja estrutura compreende medidas de exposições por grupos ocupacionais, estimadas a cada triênio, com as respectivas estimativas de prevalências específicas na Finlândia 6 . Essa MEO foi empregada para calcular estimativas em outros países como a Austrália 7, e para o benzeno, especificamente, na cidade de Montreal, Canadá 8 , na Espanha 9, em sete países da União Europeia 10, e outros países nórdicos 11. Estudos de viabilidade e reprodutibilidade da FINJEM conduzidos em diferentes contextos mostraram resultados considerados como aceitáveis 8,12.

Empregando-se a FINJEM, a estimativa de prevalência da exposição ocupacional ao benze- no na Finlândia, em 1950, foi de $0,5 \%$, que se reduziu para < 0,1\% em 2008 5. Em Montreal, entre 1945 e 1995, a prevalência média foi 0,4\% 8 . Na Espanha, em um grupamento menor de ocupações do que os listados na FINJEM, a prevalência foi estimada em 0,2\% 9. Em um estudo de vigilância de base populacional realizado na Austrália, com percepções de especialistas sobre exposição ao benzeno para diferentes grupos ocupacionais, estimou-se em $10 \%$ a prevalência de exposição ao benzeno em trabalhadores do sexo masculino, entre 2011 e 2012 13. Não foram encontrados estudos com estimativas de prevalência de exposição ao benzeno entre trabalhadores no Brasil Em uma busca sistemática de dados sobre o benzeno para a criação de uma MEO de base nacional, nenhum sistema de informação ou registros de mensurações quantitativas foi encontrado, com livre acesso para a pesquisa 14 . Neste estudo, estima-se o número de trabalhadores expostos $\mathrm{e}$ a prevalência da exposição ocupacional ao benzeno, empregando-se parâmetros da FINJEM, aplicados para o Brasil, no ano de 2010.

\section{Métodos}

Este é um estudo cuja população de referência foi o total de trabalhadores ativos ocupados, distribuído por grupos da Classificação de Ocupações para Pesquisas Domiciliares (COD. Instituto Brasileiro de Geografia e Estatística. http://www. ibge.gov.br/concla/posocupacoes/ibgexcbo94. xls, acessado em 15/Fev/2013). A COD é empregada pelo Instituto Brasileiro de Geografia e Estatística (IBGE) em inquéritos residenciais como o do Censo Demográfico de 2010 (http://www.ib ge.gov.br, acessado em Fev/2013), e se baseia na International Standard Classification of Occupations (ISCO-88) 15, da Organização Internacional do Trabalho (OIT), mantendo-se idêntica para os grandes grupos, podendo agregar subgrupos para atender a necessidades locais. Especificamente, foram obtidas planilhas com o número de trabalhadores ativos e ocupados por grupo de ocupação da COD, de acordo com o sexo. Os dados sobre a exposição ao benzeno provêm da FINJEM 16, que emprega medidas ambientais de benzeno no ar, obtidas em diferentes postos de trabalho de empresas, nas quais se conhecia que pelo menos $5 \%$ dos trabalhadores estavam expostos a um mínimo de 0,1ppm de média anual. Ou seja, esses trabalhadores eram elegíveis para a vigilância do benzeno por meio de mensurações ambientais. Essas mensurações estão disponíveis para diferentes períodos de tempo. Neste estudo, empregaram-se os dados de 1960 a 1984 , período em que a base era a mais completa, com 
o maior número de grupos ocupacionais com medidas aferidas. $\mathrm{O}$ acesso à FINJEM requer solicitação ao FIOH.

\section{Definição de variáveis}

Grupos ocupacionais potencialmente expostos ao benzeno no trabalho correspondem aos identificados pela FINJEM como elegíveis para o monitoramento do benzeno, segundo critérios citados anteriormente: (1) Químicos; (2) Assistentes de Laboratório; (3) Frentistas de Postos de Combustíveis; (4) Tapeceiros/Estofadores e afins; (5) Trabalhadores do Tratamento de Couros e Peles; (6) Sapateiros e afins; (7) Operadores de Máquinas de Processar Couros e Peles; (8) Trabalhadores da Fabricação de Calçados; (9) Operadores e Mecânicos de Máquinas e Motores; (10) Pintores e afins; (11) Impressores; (12) Trabalhadores da Impressão Gráfica; (13) Trabalhadores de Instalações de Processos Químicos; (14) Trabalhadores de Refinaria e da Indústria Química; (15) Operadores de Máquinas de Fabricar Borracha; e (16) Operadores de Máquinas de Lavar, Tingir e Passar. Para fins da análise, acrescentou-se o grupo Outros (17) de não potencialmente expostos ao benzeno, para acomodar os demais grupos ocupacionais. A outra variável, número de trabalhadores expostos ao benzeno ocupacional, deriva da aplicação das estimativas de prevalências de exposição que compõem a FINJEM, específicas por grupo ocupacional, ao número de trabalhadores correspondentes no Brasil, sendo, portanto, contínua. Sexo foi variável descritora.

\section{Análise de dados}

Os grupos ocupacionais da COD, códigos de quatro dígitos, empregados no Censo de 2010, foram agregados para se garantir equivalência aos utilizados na FINJEM. Como não havia correspondência perfeita entre grupamentos dessas duas fontes de dados, foi necessário realizar ajustes para alguns grupos da FINJEM que eram inexistentes na COD ou vice-versa. Essa correspondência de grupamentos ocupacionais da COD e FINJEM teve sua aceitação avaliada por cinco especialistas em segurança e saúde do trabalho, com experiência de estudos e inspeções ambientais relacionados à exposição ocupacional ao benzeno. A cada uma das combinações atribuíram-se graus de acordo/desacordo, empregando uma escala de 0 a 4: 0 = discordo completamente; 1 = discordo pouco; 2 = concordo; 3 = concordo muito; e 4 = concordo completamente. Estimaram-se proporções para cada combinação de grupos ocupacionais.
A prevalência de exposição potencial ao benzeno foi calculada dividindo-se a soma do número de trabalhadores dos grupos ocupacionais potencialmente expostos pelo total de trabalhadores no país. Enquanto a prevalência de exposição ao benzeno corresponde à divisão entre o somatório do número esperado de trabalhadores expostos, com base na prevalência estimada na FINJEM por cada grupamento ocupacional, pelo total de trabalhadores. As análises foram conduzidas empregando-se os aplicativos SAS 9.2 (SAS Inst., Cary, Estados Unidos) e o Excel (Microsoft Corp., Estados Unidos). O protocolo do estudo foi registrado no Sistema Nacional de Ética em Pesquisa (SISNEP), e aprovado pelo Comitê de Ética em Pesquisa do Instituto de Saúde Coletiva da Universidade Federal da Bahia (processo no 17268313.8.0000.5030). Trata-se de dados secundários e de bases agregadas que não permitem a identificação de sujeitos.

\section{Resultados}

No Brasil, foram identificados 86.353.839 trabalhadores ativos e ocupados no Censo de 2010. Dos 16 grupos ocupacionais considerados potencialmente expostos ao benzeno no trabalho, somam-se 7.376.761 trabalhadores, correspondendo a uma prevalência de $8,5 \%$. Dentre os potencialmente expostos, estimou-se um total de 770.212 trabalhadores expostos ao benzeno no trabalho e uma prevalência ponderada de $0,9 \%$ (Tabela 1). O grupo ocupacional com o maior número de expostos foi o de Operadores e Mecânicos de Máquinas e Motores $(n=4.741 .156)$, que representou mais da metade do total $(61,6 \%)$, seguido pelos Frentistas de Postos de Combustíveis ( $n=129.313$ ) com $16,8 \%$ dos expostos.

Resultados específicos por sexo são mostrados na Tabela 2, na qual se verifica que dentre os trabalhadores do sexo masculino foram estimados 552.147 expostos ao benzeno, correspondendo à prevalência ponderada de 1,1\%. Entre os expostos, a maioria era de Operadores e Mecânicos de Máquinas e Motores ( $\mathrm{n}=318.313 ; 57,7 \%$ ) compostos, por sua vez, mais comumente por Mecânicos e Reparadores de Veículos a Motor ( $n=84.393 ; 27 \%$ ) e Operadores de Instalações Fixas e Máquinas ( $\mathrm{n}=80.757 ; 25,4 \%$ ). Vale mencionar que outro grupo de grande concentração de trabalhadores expostos foi o de Frentistas de Postos de Combustíveis ( $\mathrm{n}=112.244,20,3 \%$ ), destacando-se também os Pintores e Afins $(n=33.134$; $6 \%$ ). Ainda na Tabela 2, verifica-se que entre as mulheres foi calculado que 218.065 trabalhadoras estavam expostas ao benzeno ocupacional, com maioria também no grupo de Operadoras e 
Tabela 1

Estimativa do número de trabalhadores com exposição ao benzeno no trabalho. Brasil, 2010.

\begin{tabular}{|c|c|c|c|c|}
\hline \multirow[t]{2}{*}{ Grupamentos ocupacionais por exposição potencial ao benzeno } & \multirow{2}{*}{$\begin{array}{c}\text { Prevalência da } \\
\text { exposição ao } \\
\text { benzeno * } \\
\%\end{array}$} & \multirow{2}{*}{$\begin{array}{l}\text { Trabalhadores } \\
\text { ativos e } \\
\text { ocupados no } \\
\text { Brasil ** } \\
\text { n }\end{array}$} & \multicolumn{2}{|c|}{$\begin{array}{c}\text { Expostos ao benzeno no } \\
\text { trabalho no Brasil }\end{array}$} \\
\hline & & & $\mathbf{n}$ & $\%$ \\
\hline Todos & - & 86.353 .839 & 770.212 & 0,9 \\
\hline Grupamentos ocupacionais não expostos ao benzeno & - & 78.977 .078 & 0 & - \\
\hline Grupamentos ocupacionais potencialmente expostos ao benzeno & & 7.376 .761 & 770.212 & 100,0 \\
\hline Químicos & 7,0 & 123.556 & 8.649 & 1,1 \\
\hline Assistentes de Laboratório & 8,0 & 193.081 & 15.446 & 2,0 \\
\hline Frentistas de Posto de Combustíveis & 70,0 & 184.733 & 129.313 & 16,8 \\
\hline Tapeceiros/Estofadores e afins & 3,0 & 239.397 & 7.182 & 0,9 \\
\hline Trabalhadores do Tratamento de Couros e Peles & 5,0 & 17.361 & 868 & 0,1 \\
\hline Sapateiros e afins & 10,0 & 136.070 & 13.607 & 1,8 \\
\hline Operadores de Máquina de Processar Couros e Peles & 15,0 & 3.509 & 526 & 0,1 \\
\hline Trabalhadores da Fabricação de Calçados & 15,0 & 99.719 & 14.958 & 1,9 \\
\hline Operadores e Mecânicos de Máquinas e Motores & 10,0 & 4.741 .156 & 474.116 & 61,6 \\
\hline Engenheiros mecânicos & 10,0 & 58.299 & 5.830 & 1,2 \\
\hline Técnicos em Engenharia Mecânica & 10,0 & 158.986 & 15.899 & 3,4 \\
\hline Mecânicos de Sistemas de Refrigeração/Climatização & 10,0 & 28.458 & 2.846 & 0,6 \\
\hline Mecânicos e Reparadores de Veículos a Motor & 10,0 & 871.291 & 87.129 & 18,4 \\
\hline Mecânicos e Reparadores de Motores e Máquinas & 10,0 & 383.383 & 38.338 & 8,1 \\
\hline Operadores de Máquinas de Lavrar Madeira & 10,0 & 56.213 & 5.621 & 1,2 \\
\hline Operadores de Máquinas de Costura & 10,0 & 1.462 .066 & 146.207 & 30,8 \\
\hline Operadores de Instalações Fixas e Máquinas & 10,0 & 965.237 & 96.524 & 20,4 \\
\hline Mecânicos e Montadores de Maquinaria & 10,0 & 311.542 & 31.154 & 6,6 \\
\hline Operadores de Máquinas Agrícolas & 10,0 & 445.681 & 44.568 & 9,4 \\
\hline Pintores e afins & 5,0 & 685.172 & 34.259 & 4,4 \\
\hline Impressores & 5,0 & 108.287 & 5.414 & 0,7 \\
\hline Trabalhadores de Impressão Gráfica & 5,0 & 42.860 & 2.143 & 0,3 \\
\hline Trabalhadores de Instalações de Processos Químicos & 10,0 & 356.568 & 35.657 & 4,6 \\
\hline Trabalhadores de Refinaria e da Indústria Química & 8,0 & 248.316 & 19.865 & 2,6 \\
\hline Operadores de Máquinas de Fabricar Borracha & 5,0 & 114.985 & 5.749 & 0,7 \\
\hline Operadores de Máquinas de Lavar, Tingir e Passar & 3,0 & 81.991 & 2.460 & 0,3 \\
\hline
\end{tabular}

* Dados da Finnish National Job-Exposure Matrix (Finnish Institute of Occupational Health 6);

** Dados do Censo Demográfico de 2010 (Instituto Brasileiro de Geografia e Estatística. http://www.ibge.gov.br, acessado em Fev/2013) .

Mecânicas de Máquinas e Motores ( $\mathrm{n}=155.803$; $71,4 \%)$. Mas, distintamente dos trabalhadores do sexo masculino, este grupo se constituiu quase exclusivamente de Operadoras de Máquinas de Costura ( $n=128.132 ; 82,2 \%$ ). Deve-se destacar que semelhantemente aos homens, o segundo grupo de maior contribuição para os expostos foi o de mulheres que trabalhavam como Frentistas de Postos de Combustíveis ( $\mathrm{n}=17.069$; 7,8\%), e Trabalhadoras de Instalações de Processos Químicos ( $n=12.820 ; 5,9 \%$ ). No total, a razão de sexos do número de prováveis expostos ao benzeno no trabalho foi de 2,5 homens para cada mulher, com perfis muito distintos entre os grupamentos ocupacionais.

Os 64 pares de grupamentos ocupacionais COD Brasil e FINJEM são mostrados na Tabela 3. Do total, 30 pares (47\%) receberam o escore de "concordo completamente" (3 ou 4) dos especialistas; incluindo-se os pares que receberam "concordo moderadamente", a aceitação da correspondência atribuída alcançou 97\%; os grupamentos ocupacionais dos pares restantes que receberam "desacordos" foram redistribuídos 
Número de expostos e prevalência de exposição ao benzeno no trabalho por grupamentos ocupacionais, de acordo com o sexo. Brasil, 2010.

\begin{tabular}{|c|c|c|c|c|c|c|}
\hline \multirow{3}{*}{$\begin{array}{l}\text { Grupamentos ocupacionais por exposição } \\
\text { potencial ao benzeno }\end{array}$} & \multicolumn{3}{|c|}{ Homens } & \multicolumn{3}{|c|}{ Mulheres } \\
\hline & \multirow{2}{*}{$\begin{array}{c}\text { Ativos } \\
\text { ocupados } \\
\text { n }\end{array}$} & \multicolumn{2}{|c|}{$\begin{array}{l}\text { Expostos ao benzeno no } \\
\text { trabalho }\end{array}$} & \multirow{2}{*}{$\begin{array}{c}\text { Ativos } \\
\text { ocupados } \\
\text { n }\end{array}$} & \multicolumn{2}{|c|}{$\begin{array}{c}\text { Expostos ao benzeno no } \\
\text { trabalho }\end{array}$} \\
\hline & & $\mathbf{n}$ & $\%$ & & $\mathbf{n}$ & $\%$ \\
\hline Total & 49.823 .312 & 552.147 & 1,1 & 36.530 .527 & 218.065 & 0,6 \\
\hline Grupamentos ocupacionais não expostos ao benzeno & 44.642 .178 & 0 & - & 34.334 .900 & 0 & - \\
\hline Grupamentos ocupacionais expostos ao benzeno & 5.181 .134 & 552.147 & 100,0 & 2.195 .627 & 218.065 & 100,0 \\
\hline Químicos & 86.705 & 6.069 & 1,1 & 36.851 & 2.580 & 1,2 \\
\hline Assistentes de Laboratório & 114.768 & 9.181 & 1,7 & 78.313 & 6.265 & 2,9 \\
\hline Frentistas de Posto de Combustíveis & 160.349 & 112.244 & 20,3 & 24.384 & 17.069 & 7,8 \\
\hline Tapeceiros/Estofadores e afins & 113.847 & 3.415 & 0,6 & 125.550 & 3767 & 1,7 \\
\hline Trabalhadores do Tratamento de Couros e Peles & 13.377 & 669 & 1,1 & 3.984 & 199 & 0,1 \\
\hline Sapateiros e afins & 80.929 & 8.093 & 1,5 & 55.141 & 5.514 & 2,5 \\
\hline Operadores de Máquinas de Processar Couros e & 3.104 & 466 & 0,1 & 405 & 61 & 0,0 \\
\hline \multicolumn{7}{|l|}{ Peles } \\
\hline Trabalhadores da Fabricação de Calçados & 53.150 & 7.973 & 1,4 & 46.569 & 6.985 & 3,2 \\
\hline Operadores e Mecânicos de Máquinas e Motores & 3.183 .131 & 318.313 & 57,7 & 1.558 .025 & 155.803 & 71,4 \\
\hline Engenheiros Mecânicos & 54.624 & 5.462 & 1,7 & 3.675 & 368 & 0,2 \\
\hline Técnicos em Engenharia Mecânica & 152.000 & 15.200 & 4,8 & 6.986 & 699 & 0,4 \\
\hline Mecânicos de Sistemas Refrigeração/Climatização & 27.309 & 2.731 & 0,8 & 1.149 & 115 & 0,1 \\
\hline Mecânicos e Reparadores de Veículos a Motor & 843.932 & 84.393 & 26,5 & 27.359 & 2.736 & 1,8 \\
\hline Mecânicos e Reparadores de Motores e Máquinas & 366.324 & 36.632 & 11,5 & 17.059 & 1.706 & 1,1 \\
\hline Operadores de Máquinas de Lavrar Madeira & 46.891 & 4.689 & 1,5 & 9.322 & 932 & 0,6 \\
\hline Operadores de Máquinas de Costura & 180.746 & 18.075 & 5,7 & 1.281 .320 & 128.132 & 82,2 \\
\hline Operadores de Instalações Fixas e Máquinas & 807.569 & 80.757 & 25,4 & 157.668 & 15.767 & 10,1 \\
\hline Mecânicos Montadores e Maquinaria & 275.117 & 27.512 & 8,6 & 36.425 & 3.643 & 2,3 \\
\hline Operadores de Máquinas Agrícolas & 428.619 & 42.862 & 13,5 & 17.062 & 1.706 & 1,1 \\
\hline Pintores e afins & 662.677 & 33.134 & 6,0 & 22.495 & 1.125 & 0,5 \\
\hline Impressores & 95.244 & 4.762 & 0,9 & 13.043 & 652 & 0,3 \\
\hline Trabalhadores de Impressão Gráfica & 27.987 & 1.399 & 0,3 & 14.873 & 744 & 0,3 \\
\hline Trabalhadores de Instalações de Processos & 228.373 & 22.837 & 4,1 & 128.195 & 12.820 & 5,9 \\
\hline \multicolumn{7}{|l|}{ Químicos } \\
\hline Trabalhadores de Refinaria e da Indústria Química & 213.158 & 17.053 & 3,1 & 35.158 & 2.813 & 1,3 \\
\hline Operadores de Máquinas de Fabricar Borracha & 110.407 & 5.520 & 1,0 & 4.578 & 229 & 0,1 \\
\hline Operadores de Máquinas de Lavar, Tingir e Passar & 33.928 & 1.018 & 0,2 & 48.063 & 1.442 & 0,7 \\
\hline
\end{tabular}

Fonte: Censo Demográfico de 2010 (Instituto Brasileiro de Geografia e Estatística. http://www.ibge.gov.br, acessado em Fev/2013) e dados da Finnish Institute of Occupational Health 6 .

segundo propostas dos próprios especialistas participantes.

\section{Discussão}

No Brasil, em 2010, estima-se que 7.376.761 trabalhadores desenvolviam atividades de trabalho nas quais podiam entrar em contato com o benzeno, que denominamos exposição potencial a essa substância. Dentre eles, 770.212 trabalha- dores podem ser considerados como expostos ao benzeno no trabalho, o que corresponde a uma prevalência ponderada por grupos ocupacionais de $0,9 \%$ de todos os trabalhadores do país. Maiores estimativas de prevalência de exposição ocupacional ao benzeno foram encontradas entre os homens quando comparados às mulheres. Essa exposição se concentrava nos grupos ocupacionais de Operadores e Mecânicos de Máquinas e Motores e Frentistas de Postos de Combustíveis, tanto em homens quanto em mulheres. Entre as 
Tabela 3

Correspondência dos grupos ocupacionais da Finnish National Job-Exposure Matrix (FINJEM) e da Classificação de Ocupações para Pesquisas Domiciliares (COD), do Censo Demográfico de 2010, no Brasil.

\begin{tabular}{|c|c|c|c|c|}
\hline & FINJEM & & COD & Observação \\
\hline Código & Título do grupo & Código & Título do grupo & \\
\hline \multirow[t]{6}{*}{20} & Chemists & 2113 & Químicos & \\
\hline & & 2145 & Engenheiros Químicos & \\
\hline & & 3111 & Técnicos em Ciências Físicas e Químicas & \\
\hline & & 3133 & Controladores de Instalações de Processamento de Produtos & Corresponde à COD FINJEM 731 \\
\hline & & & Químicos & \\
\hline & & 8131 & Operadores de Instalações e Máquinas de Produtos Químicos & \\
\hline \multirow[t]{7}{*}{028} & Laboratory Assistants & 3116 & Técnicos de Química Industrial & Corresponde à COD FINJEM 736 \\
\hline & & 3141 & Técnicos de Nível Médio de Biologia & \\
\hline & & 3142 & Técnicos de Agropecuários e Florestais & \\
\hline & & 3212 & Técnicos de Laboratórios Médicos & \\
\hline & & 3213 & Técnicos e Assistentes Farmacêuticos & \\
\hline & & 3214 & Técnicos de Próteses Médicas e Dentárias & \\
\hline & & 3240 & Técnicos de Assistentes Veterinários & \\
\hline 234 & Service Station Attendants & 5245 & Frentistas de Posto de Combustíveis & \\
\hline 613 & Upholsterers & 7534 & Tapeceiros, Colchoeiros e afins & \\
\hline \multirow[t]{2}{*}{621} & Leather Cutters for & 7535 & Trabalhadores da Indústria de Couros e Peles & \\
\hline & Footwear & 8159 & Operadores de Máquinas Têxteis, Couro e Pele não Classificados & \\
\hline 622 & Shoe Sewers & 7536 & Sapateiros e afins & \\
\hline 623 & $\begin{array}{l}\text { Lasters and Sole Fitters, } \\
\text { etc. }\end{array}$ & 8155 & Operadores de Máquinas de Processamento de Couro e Peles & \\
\hline 624 & Footwear Workers, etc. & 8156 & Operadores de Máquinas na Fabricação de Calçados e afins & \\
\hline \multirow[t]{12}{*}{652} & Machine and Engine & 2144 & Engenheiros Mecânicos & \\
\hline & Mechanics & 3115 & Técnicos em Engenharia Mecânica & \\
\hline & & 3155 & Técnicos em Segurança Aeronáutica & \\
\hline & & 7127 & Mecânicos de Refrigeração & \\
\hline & & 7231 & Mecânicos e Reparadores de Veículos a Motor & \\
\hline & & 7232 & Mecânicos e Reparadores de Motores de Avião & \\
\hline & & 7233 & Mecânicos e Reparadores de Máquinas Agrícolas Industriais & \\
\hline & & 7234 & Reparadores de Bicicletas e afins & \\
\hline & & 7523 & Operadores de Máquinas de Lavrar Madeira & \\
\hline & & 81 & Operadores de Instalações Fixas e Máquinas & $\begin{array}{c}\text { Exceto } 8155,8156,8159,8131 \\
8141 \text { e } 8157 \text { já classificados }\end{array}$ \\
\hline & & 82 & Montadores & \\
\hline & & 83 & Condutores de Veículos e Operadores de Equipamentos & Exceto 835 \\
\hline 680 & $\begin{array}{l}\text { Painters, lacquerers and } \\
\text { floor layers }\end{array}$ & 713 & Pintores, Limpadores de Fachada e afins & \\
\hline 701 & Printers & 7322 & Impressores & \\
\hline 709 & $\begin{array}{l}\text { Occupation in graphics, } \\
\mathrm{Nec}\end{array}$ & 732 & Trabalhadores da Impressão Gráfica & $\begin{array}{l}\text { Exceto o código } 7322 \text { - } \\
\text { corresponde à COD FINJEM } 736\end{array}$ \\
\hline 730 & Distillers & 3134 & Operadores de Instalações de Refino de Petróleo e Gás Natural & Classificado à COD FINJEM 736 \\
\hline \multirow[t]{2}{*}{731} & Cookers, furnacemen & 3133 & Controladores de Instalação de Processamento de Produtos & \\
\hline & (chemical processes) & & Químicos & \\
\hline 736 & $\begin{array}{l}\text { Refinery loccupations in } \\
\text { chemical industry }\end{array}$ & 3116 & Técnico em Química Industrial & \\
\hline 751 & Rubber products workers & 8.1.4.1 & Operadores de Máquinas para Fabricar Produtos de Borracha & \\
\hline 850 & Laundry workers & 8.1.5.7 & Operadores de Máquinas de Lavar, Tingir e Passar Roupas & \\
\hline
\end{tabular}

Fonte: Censo Demográfico de 2010 (Instituto Brasileiro de Geografia e Estatística. http://www.ibge.gov.br, acessado em Fev/2013) e dados da Finnish Institute of Occupational Health 6. 
mulheres expostas desse primeiro grupo ocupacional, quase a totalidade era constituída por operadoras de máquinas de costura da indústria de confecções. A correspondência entre a classificação de grupos ocupacionais empregada no Censo de 2010 e a adotada na matriz de exposição ocupacional utilizada para as nossas projeções (FINJEM) foi aceita pela maioria dos especialistas que avaliaram a sua pertinência e adequação.

Os achados deste estudo permitem inferir que a exposição ocupacional ao benzeno no Brasil é um problema de saúde pública por serem elevados o número de trabalhadores potencialmente expostos e o de expostos ao benzeno, que é um cancerígeno reconhecido pela OMS. As estimativas obtidas são apenas projeções, no entanto não surpreendem ao se considerar que o benzeno já é objeto de ações específicas de vigilância no país que, embora em implementação há mais de uma década, se ressentem da falta de estimativas da extensão populacional dessa exposição. Em que pesem os seus limites metodológicos, tais achados são fundamentais para estimativas do número de casos esperados de doenças relacionadas, como também para o estabelecimento de prioridades, e dimensionamento das atividades de prevenção e controle. Vale assinalar que estimativas nacionais somente podem ser obtidas com projeções com base em dados amostrais, ou de grupos de trabalhadores, idealmente com base em mensurações locais. Entretanto, não foram encontradas medidas de exposição ocupacional ao benzeno disponíveis para a pesquisa ou vigilância. Com base na determinação do Acordo Nacional do Benzeno, o Ministério do Trabalho e Emprego (MTE) criou um cadastro nacional composto por empresas que empregavam essse agente em seus processos produtivos. Foram listadas apenas 117 empresas, que correspondiam a 25.275 trabalhadores (Ministério do Trabalho e Emprego. Cadastro: benzeno e amianto. Empresas que trabalham com benzeno. http://trabalho.gov.br/seguranca-esaude-no-trabalho/cadastro-benzeno-e-asbes to, acessado em 17/Nov/2016) no total, mas entre os dados desse cadastro não havia estimativas de expostos ou medidas de exposição individuais ou grupais.

A prevalência estimada da exposição ocupacional para o benzeno no Brasil foi muito elevada, quase nove vezes maior que a da Finlândia $(<0,1 \%) 6$, o que reflete as diferenças dos perfis produtivos deste país em relação ao Brasil. A estimativa brasileira foi também mais de duas vezes a estimativa de Montreal $(0,4 \%)$, calculada com base na FINJEM. Contudo, a estimativa deste estudo foi mais baixa que a calculada para o Canadá, de $2 \%$ dos trabalhadores 17 , empregando-se a CAREX (CARcinogen EXposure). No Brasil, em um estudo pioneiro de 1953, estimou-se em 7,3\% a prevalência de exposição a solventes orgânicos entre os trabalhadores da indústria do Estado de São Paulo 18. Mais recentemente, observou-se que essa mesma exposição afetava 18,5\% dos trabalhadores de uma indústria gráfica no mesmo estado, no ano de 2006 19. Como já mencionado, estimativas nacionais de exposições ocupacionais a agentes químicos somente são possíveis com projeções de matrizes de exposições ocupacionais, um recurso metodológico ainda pouco utilizado e conhecido pelos órgãos responsáveis pela vigilância, e mesmo para pesquisas no Brasil. O uso desse recurso pode enfrentar resistências por conta da imprecisão das medidas resultantes, porém é a única estratégia possível para se obter indicadores nacionais de exposições ocupacionais, informação fundamental para a vigilância em saúde do trabalhador.

Os achados deste trabalho evidenciam que a maior proporção estimada de trabalhadores expostos ao benzeno foi a do grupo de Operadores e Mecânicos de Máquinas e Motores (62\%). Suas atividades comumente envolvem a reparação de máquinas e veículos, em que o uso do benzeno como solvente, diluente, ou desengordurante para a limpeza de peças 20 é frequente, todavia elas não são contempladas por normas de segurança e de saúde específicas no Brasil. Ademais, esses operadores e mecânicos são conhecidos por manipularem equipamentos aquecidos, que intensificam a liberação de vapores de benzeno, empregado na lubrificação de peças ou como combustível 21,22. O benzeno é substância comum nos óleos básicos, que compõem lubrificantes extensamente utilizados na operação de máquinas e motores. A maior concentração de expostos entre os Operadores e Mecânicos de Máquinas e Motores também foi encontrada no Canadá, em 2013 17. Na Itália, para o período de 1996 a 2007 23, esse mesmo grupo foi o 2 o com maior número de expostos ao benzeno, antecedido apenas pelos motoristas de caminhões e veículos pesados. Neste estudo, Frentistas de Postos de Combustíveis foi o segundo grupo com maior número de trabalhadores expostos ao benzeno no Brasil, onde essa ocupação é muito comum. Isso ocorre porque o abastecimento de veículos de transporte é realizado manualmente, distintamente de outros países, onde a atividade é responsabilidade do consumidor, parcial ou totalmente, além de se empregarem sistemas fechados de captação e recuperação de vapores no abastecimento, carregamento e descarregamento de combustíveis 24 . Frentistas entram em contato com o benzeno: pela via dérmica, durante o abastecimento ao manipularem tampas dos tanques de combustí- 
vel de veículos, mangueiras, e ao enxugarem derramamentos; pela via respiratória, ao entrarem em contato com vapores, não apenas dos tanques do veículo em abastecimento, mas das emissões dos escapamentos, cujo benzeno resulta da combustão incompleta do tolueno e xileno 25 . Além disso, outra fonte de exposição de benzeno para frentistas são as emissões dos tanques de armazenamento subterrâneo, especialmente quando estão sendo abastecidos 26 . Por não serem do ramo manufatureiro, os frentistas não foram originalmente contemplados no Acordo Nacional do Benzeno, o que levou a um movimento que resultou na sua incorporação como grupo alvo, à semelhança do que ocorreu no protocolo de risco químico, do Ministério da Saúde 27.

Maiores prevalências de exposição ocupacional ao benzeno também foram encontradas entre os homens, no Canadá, em 2013 17. Essa diferença é esperada devido à concentração do sexo masculino em atividades industriais - em que prevalece a exposição ao benzeno - e em atividades de serviços, os frentistas. Vale ressaltar que, distintamente, no grupo de Operadores e Mecânicos de Máquinas e Motores, o subgrupo de operadores de máquinas de costuras foi quase totalmente constituído por mulheres. Isso pode ser decorrente do universo de trabalhadores ativos, formais e informais, contemplado neste estudo, que incluiu um grande contingente de trabalhadoras da indústria da confecção de pequeno porte e não registrada. Este resultado é consistente com os encontrados em um estudo conduzido com dados de mensuração do benzeno no ar, na China, que evidenciaram níveis elevados de exposição ocupacional a essa substância, em ambientes de trabalho compartilhados por costureiras 28 .

Neste estudo empregou-se a COD, cuja estrutura tem como referência a ISCO-88 15, também utilizada na FINJEM. Apesar da referência comum, foram identificadas diferenças que precisaram ser ajustadas. Para tal, entre os grupos ocupacionais empregados na COD e FINJEM, foram estabelecidas correspondências avaliadas por especialistas que as consideraram aceitáveis, na sua maioria. Isso fortaleceu as conclusões do estudo, no que tange à redução de erros de classificação, ao contribuir para maior similaridade entre os grupamentos respectivos de ambas as classificações.

Todavia, as conclusões deste estudo devem ser consideradas com cautela. Em primeiro lugar, empregou-se a FINJEM, construída com dados de empresas da Finlândia, país no qual o perfil produtivo, o nível de cobertura e a qualidade da proteção à saúde e segurança do trabalhador são muito distintos do Brasil. Entretanto, não foram encontradas medidas de exposição ao benzeno em ambientes de trabalho disponíveis para investigação ou para construção de uma MEO com dados locais que permitisse a projeção do número de expostos. A Finlândia já dispunha, à época da coleta de dados empregados neste estudo, de um dos melhores sistemas de proteção à segurança e saúde dos trabalhadores no mundo 2, diferentemente do Brasil 29. Essa diferença pode ter sido minimizada, considerando-se que os dados da FINJEM foram de 1960 a 1984, quando o total de grupos ocupacionais elegíveis contava com medidas da exposição, enquanto os dados brasileiros se referem a 2010. Isso pode ter produzido imprecisões em nossas estimativas, mas diferenças entre os estágios de desenvolvimento tecnológico da indústria, como também da qualidade do treinamento, do nível de qualificação, organização e engajamento dos trabalhadores na luta por ambientes de trabalho seguros e saudáveis, entre os dois países, devem ser lembradas. Esses fatores influenciam o cumprimento de parte das empresas das normas de segurança e proteção à saúde, reconhecido como principal determinante da exposição ao benzeno. Outro limite do estudo foi o uso da COD, que é própria para estudos domiciliares conduzidos em inquéritos populacionais, e não para investigações em saúde do trabalhador, nas quais grupos ocupacionais são utilizados para compreender perfis de exposição, podendo ser até mesmo considerados como grupos homogêneos de exposição. Vale ressaltar que os dados da FINJEM refletem exposições médias de 0,1ppm ao ano, mas estudos recentes 30,31 revelam que valores menores podem ter também efeitos sobre a saúde. Infelizmente, estimativas da exposição ao benzeno para ocupações reconhecidas como potencialmente expostas ao benzeno, como bombeiros e motoristas de cargas perigosas, não estavam disponíveis na FINJEM, impedindo a sua incorporação na análise deste estudo. Além disso, utilizaram-se apenas grupamentos ocupacionais, quando o desejável seria a desagregação desses grupamentos pelos diferentes ramos de atividade econômica, que poderia aumentar a precisão das estimativas.

Este estudo avança no conhecimento epidemiológico sobre um importante cancerígeno químico, largamente empregado na indústria brasileira, para o qual existe uma política específica de controle e de proteção dos trabalhadores, que recomenda o monitoramento da exposição e dos efeitos sobre a saúde, mas que tem falhado em produzir e divulgar dados mínimos sobre a magnitude e distribuição em território nacional. Mesmo com os limites apontados, pode-se dimensionar o número de trabalhadores potencialmente e expostos ao benzeno no trabalho, 
sua distribuição entre os grupos ocupacionais, com base no perfil produtivo do país. Matrizes de exposição ocupacional são instrumentos amplamente reconhecidos por sua utilidade no estabelecimento de projeções populacionais, fundamentais à Saúde Coletiva, especialmente na definição de prioridades para as ações de prevenção e proteção dos trabalhadores. Por essas características são empregadas, mesmo em países que contam com mensurações de exposição, mas que dificilmente atingem o total de trabalhadores por sua inviabilidade operacional. Não obstante, MEO vêm sendo utilizadas em países diferentes daqueles de onde se originaram os dados, a exemplo da FINJEM 8,9,10,11, também empregada como referência na construção de outras matrizes 10,11.

Este estudo demonstra a falta de acesso público e transparência de dados de empresas do setor de produção, distribuição e comércio relativos às mensurações quantitativas da exposição ao benzeno em ambientes de trabalho, que limitam tanto a pesquisa com dados secundários quanto o monitoramento populacional requerido na vigilância. Informações do Programa de Prevenção de Riscos Ambientais (PPRA) são produzidas pelas empresas, porém o acesso é restrito e a proposta do Sistema de Monitoramento de Populações Expostas a Agentes Químicos (SIMPEAQ) ainda se encontra insuficientemente implantada. Com esses dados seria possível realizar o necessário monitoramento temporal, sistemático e contínuo deste cancerígeno, que permitiria a produção de informações imprescindíveis à avaliação do impacto das medidas de prevenção e controle do benzeno e, portanto, ao aperfeiçoamento das atuais diretrizes de modo a garantir a saúde dos trabalhadores.

\section{Colaboradores}

M. J. M. Corrêa e V. S. Santana participaram da concepção, análise e redação do artigo.

\section{Agradecimentos}

A Timo Kauppinen que disponibilizou a Finnish $\mathrm{Na}$ tional Job-Exposure Matrix (FINJEM), e a Carlos Lessa pelo apoio no acesso aos dados do Censo Demográfico de 2010. À Coordenação Geral de Saúde do Trabalhador, Secretaria de Vigilância à Saúde, Ministério da Saúde, pelo financiamento.

\section{Referências}

1. International Agency for Research on Cancer. Monographs on the evaluation of carcinogenic risks to humans. Lyon: International Agency for Research on Cancer; 1987.

2. Rantanen J, Kauppinen T, Tiokkanen J, Kurppa K, Lehtinen S, Leino T. Work and health country profiles. Country profiles and national surveillance indicators in occupational health and safety. Helsinki: Finnish Institute of Occupational Health; 2001. (People and Work Research Reports, 44).

3. Concha-Barrientos M, Nelson DI, Driscoll T, Steenland NK, Punnett L, Fingerhut MA, et al. Selected occupational risks. In: Ezzati M, Lopez A, Rodgers A, Murray CJL, editors. Comparative quantification of health risks: global and regional burden of disease attributable to selected major risk factors. Geneva: World Health Organization; 2004. p. $1652-801$

4. Mannetje A, Kromhout H. The use of occupation and industry classifications in general population studies. Int J Epidemiol 2003; 32:419-28.

5. Kauppinen T, Uuksulainen S, Saalo A, Mäkinen I, Pukkala E. Use of the Finnish Information System on Occupational Exposure (FINJEM) in epidemiologic, surveillance, and other applications. Ann Occup Hyg 2014; 58:380-96.

6. Kauppinen T, Uuksulainen S, Saalo A, Mäkinen I. Trends of occupational exposure to chemical agents in Finland in 1950-2020. Ann Occup Hyg 2013; 57:593-607. 
7. Karipidis KK, Benke G, Sim MR, Kauppinen T, Giles G. Occupational exposure to ionizing and nonionizing radiation and risk of glioma. Occup Med 2007; 57:518-24.

8. Lavoué J, Pintos J, Van Tongeren M, Kincl L, Richardson L, Kauppinen T, et al. Comparison of exposure estimates in the Finnish Job-Exposure Matrix FINJEM with a JEM derived from expert assessments performed in Montreal. Occup Environ Med 2012; 69:465-71.

9. González-Galarzo MC, Garcia AM. Características de la exposición a riesgos laborales en distintas ocupaciones en España según las estimaciones de la Matriz Empleo-Exposición Finlandesa (FINJEM). http://www.upf.edu/cisal/_pdf/Infome_riesgos_ laborales.pdf (acessado em 12/Mar/2013).

10. Tongeren MV, Kincl L, Richardson L, Benke G, Figuerola J, Kauppinen T, et al. Assessing occupational exposure to chemicals in an international epidemiological study of brain tumors. Ann Occup Hyg 2013; 57:610-26.

11. Kauppinen T, Heikkila P, Plato N, Woldbaek T, Lenvik K, Hansen J, et al. Construction of job-exposure matrices for the Nordic Occupational Cancer Study (NOCCA). Acta Oncol 2009; 48:791-800.

12. Teschke K, Ohlsan AF, Daniels JL, De Roos AJ, Parks CG, Schulz M, et al. Occupational exposure assessment in case-control studies: opportunities for improvement. Occup Environ Med 2002; 59: 575-93.

13. Carey RN, Driscoll TR, Peters S, Glass DC, Reid A, Benle G, et al. Estimated prevalence of exposure to occupational carcinogens in Australia (2011-2012). Occup Environ Med 2013; 71:55-62.

14. Moura-Correa J, Santana VS. Matriz de exposição ocupacional potencial: usos e aplicações para estimativas populacionais de expostos ao benzeno. In: Moura-Correa MJ, Pinheiro TMM, Merlo ARC, organizadores. Vigilância em saúde do trabalhador no Sistema Único de Saúde. Belo Horizonte: Coopmed; 2013. p. 125-44.

15. International Labour Organization. International standard classification of occupations. Geneva: International Labour Organization; 1988.

16. Kauppinen T, Toikkanen J, Pukkala E. From crosstabulations to multipurpose exposure information systems: a new job-exposure matrix. Am J Ind Med 1998; 33:409-17.

17. CARcinogen EXpoxure Canada. Benzene: occupational estimate. http://www.carexcanada.ca/en/ benzene/occupational_estimate/ (acessado em 12/Mar/2013)

18. Bedrikow B, Gomes JR. Bernardo Bedrikow e a medicina do trabalho: o homem e a obra. Rev Bras Med Trab 2015; 13:43-57.

19. Dourado AG, Kato M. Avaliação da exposição ocupacional a solventes orgânicos na indústria gráfica: um estudo em 63 indústrias do estado de São Paulo. In: Dourado AG, organizador. Segurança e saúde do trabalho: uma perspectiva prática. Brasília: Unidade de Saúde e Segurança do Trabalho Departamento Nacional, Serviço Social da Indústria; 2010. p. 9-25. (Série SESI em Saúde e Segurança no Trabalho, 8).
20. Vitali M, Ensabella F, Stella D, Guidotti M. Exposure to organic solvents among handicraft car painters: a pilot study in Italy. Ind Health 2006; 44:310-7.

21. Andrade Filho A, Campolina D, Carvalho FB. Intoxicações agudas relacionadas com o trabalho. In: Mendes R, organizador. Patologia do trabalho. 3a Ed. Rio de Janeiro: Editora Atheneu; 2013. p. 1505-67.

22. Egeghy PP, Nylander-French L, Gwin KK, HertzPicciotto I, Rappaport S. Self-collected breath sampling for monitoring low-level benzene exposures among automobile mechanics. Ann Occup Hyg 2002; 46:489-500.

23. Scarselli A, Binazzi A, Marzio DD. Occupational exposure levels to benzene in Italy: findings from a national database. Occup Environ Health 2011; 84:617-25.

24. Szwarc A, Farah EL, Branco GM, Branco FC. Redução da emissão evaporativa do veículo em movimento e no reabastecimento de combustível. In: XXII Simpósio Internacional de Engenharia Automotiva; Blucher Engineering Proceedings. http:// pdf.blucher.com.br/engineeringproceedings/ simea2014/38.pdf (acessado em Ago/2014).

25. US Environmental Protection Agency. Air toxics from motor vehicles. http:www.epa.gov/otaq/ consumer/02-toxic.pdf (acessado em 20/Jul/ 2015).

26. Guldberg PH. Gasoline and vapor exposures in service station and leaking underground storage tank scenarios. J Expo Anal Environ Epidemiol 1992; 2:97-107.

27. Departamento de Ações Programáticas Estratégicas, Secretaria de Atenção à Saúde, Ministério da Saúde. Risco químico: atenção à saúde dos trabalhadores expostos ao benzeno. Brasília: Editora MS; 2006. (Saúde do Trabalhador, 7. Protocolos de Complexidade Diferenciada) (Série A. Normas e Manuais Técnicos).

28. Waidyanatha S, Rothman N, Li G, Smith MT, Yin S, Rappaport M. Rapid determination of six urinary benzene metabolites in occupationally exposed and unexposed subjects. Anal Biochem 2004; 327:184-99.

29. Santana VS, Dias EC, Silva JFS. Atenção, prevenção e controle em saúde do trabalhador. In: Paim JS, Almeida Filho ND, organizadores. Saúde coletiva: teoria e prática. Rio de Janeiro: MedBook; 2014. p. 513-39.

30. Rappaport SM, Kim S, Lan Q, Vermeulen R, Waidyanatha S, Zhang L, et al. Evidence that humans metabolize benzene via two pathways. Environ Health Perspect 2009; 117:946-52.

31. Smith MT. Advances in understanding benzene health effects and susceptibility. Annu Rev Public Health 2010; 31:133-48. 


\section{Abstract}

This study estimates the number of exposed workers and the prevalence of occupational benzene exposure in Brazil. Due to the lack of available local measurements for the study, data were used from an occupational exposure matrix, the Finnish National JobExposure Matrix (FINJEM), which covers proportions of individuals exposed to benzene, calculated as environmental measures. In Brazil, the 2010 Demographic Census identified 86,353,839 workers in the workforce and employed. Applying the FINJEM parameters, an estimated 7,376,761 (8.5\%) belonged to potentially exposed occupational groups, while 770,212 were considered exposed to benzene, corresponding to an occupational group-weighted prevalence of $0.9 \%$, higher in men (1.1\%) than in women (0.6\%). Exposed individuals were concentrated in the category of Machine and Motor Operators and Mechanics (62\%). The number of exposed and prevalence of occupational exposure to benzene are high, even when compared to Finnish parameters, suggesting the need for monitoring and control of this carcinogen in Brazil.

Occupational Exposure; Benzene; Occupational Health

\section{Resumen}

En este trabajo se estima el número de trabajadores expuestos y la prevalencia de la exposición ocupacional al benceno en Brasil. Debido a la falta de mediciones locales disponibles para esta investigación, se emplearon datos de una matriz de exposición ocupacional: la Finnish National Job-Exposure Matrix (FINJEM), que contempla proporciones de expuestos al benceno, calculadas con medidas ambientales. En Brasil, el Censo Demográfico de 2010 identificó a 86.353.839 trabajadores activos y ocupados. Aplicándose los parámetros de la FINJEM, se estimó que 7.376 .761 (8,5\%) pertenecían a grupos ocupacionales potencialmente expuestos, mientras que 770.212 fueron considerados expuestos al benceno, correspondiendo a una prevalencia ponderada por grupo ocupacional de 0,9\%, mayor en hombres $(1,1 \%)$ que en mujeres $(0,6 \%)$. Los expuestos se concentraron en el grupo de Operadores y Mecánicos de Máquinas y Motores (62\%). El número de expuestos y la prevalencia de la exposición ocupacional al benceno son elevados, incluso considerando parámetros de Finlandia, sugiriendo la necesidad de un monitoreo y control de ese cancerígeno en Brasil.

Exposición Profesional; Benzeno; Salud Laboral
Recebido em 14/Ago/2016

Versão final reapresentada em 17/Jan/2016 Aprovado em 06/Abr/2016 